\title{
Kozai resonance in extrasolar systems
}

\author{
A.-S. Libert ${ }^{1,2, \star}$ and K. Tsiganis ${ }^{2, \star \star}$ \\ 1 Department of Mathematics FUNDP, 8 Rempart de la Vierge, 5000 Namur, Belgium \\ e-mail: anne-sophie.libert@fundp.ac.be; tsiganis@astro.auth.gr \\ 2 Section of Astrophysics Astronomy and Mechanics, Department of Physics, University of Thessaloniki, \\ 54 124, Thessaloniki, Greece
}

Received 21 August 2008 / Accepted 13 October 2008

\begin{abstract}
Aims. We study the possibility that extrasolar two-planet systems, similar to the ones that are observed, can be in a stable Kozairesonant state, assuming a mutual inclination of the orbital planes of order $I_{\text {mut }} \sim 40-60^{\circ}$.

Methods. Five known multi-planet systems that are not in mean motion resonance were selected, according to defined criteria, as "possible prototypes" ( $v$ Andromedae, HD 12661, HD 169830, HD 74156, HD 155358). We performed a parametric study, integrating several sets of orbits of the two planets, obtained by varying the (unknown) inclination of their orbital planes and their nodal longitudes, thus changing the values of their masses and mutual inclination. We also take into account the reported observational errors on the orbital elements. These numerical results are characterized using analytical secular theory and frequency analysis. Surface of section techniques are also used to distinguish between stable and chaotic motions.

Results. Frequency analysis offers a reliable way of identifying the Kozai resonance in a general reference frame, where the argument of the pericenter of the inner planet does not necessarily librate around $\pm 90^{\circ}$ as in the frame of the Laplace plane, through the noncoupling of the eccentricities of the two planets. We find that four of the five selected systems ( $v$ Andromedae, HD 12661, HD 169830 and HD 74156) could in principle be in Kozai resonance, as their eccentricities and apsidal orientations are such that the system enters in the stability region of the Kozai resonance in 20-70\% of the cases, provided that their mutual inclination is at least $45^{\circ}$. Thus, a large fraction of the observed multi-planet systems has observed orbital characteristics that are consistent with stable, Kozai-type, motion in 3D. Unstable sets of orbits are also found, due to the chaos that develops around the stability islands of the Kozai resonance. A variety of physical mechanisms that could generate the necessary large mutual inclinations are discussed, including (a) planet formation; (b) type II migration and resonant interactions during the gas-dominated phase; (c) planetesimal-driven migration and resonance crossing during the gas-free era; (d) multi-planet scattering, caused by the presence of an additional planet.
\end{abstract}

Key words. planetary systems - celestial mechanics - methods: $N$-body simulations - methods: analytical

\section{Introduction}

More than 25 extrasolar multi-planetary systems have been detected. Nonetheless the spatial resolution of their orbits is still not possible. The majority of detections are performed by the radial velocity technique, which is based on measuring only the line of sight component of the star's velocity. This results in an indetermination of some orbital parameters - the inclination of the orbital plane to the plane of the sky and the longitude of the node -, but also in a poor determination of the masses: only minimal masses can be inferred.

Because of this lack of knowledge, as well as a general belief that planetary systems would tend to resemble the solar system in being composed of co-planar orbits, only a few studies on the dynamics of the extrasolar systems have been devoted to the three-dimensional (3D) problem. Among the analytical studies of 3D exosystems that are not in mean motion resonance (MMR), are the works of Michtchenko et al. (2006) and Libert \& Henrard $(2007,2008)$. In the latter, a 12th order expansion in eccentricities and inclinations of the perturbative potential of the 3D secular three-body problem was used to study the dynamical

\footnotetext{
* FNRS Research Fellow.

$\star \star$ We wish to dedicate this paper to the memory of Jacques Henrard, a teacher and a friend, who strongly encouraged us to have this
} collaboration. features of the problem, which can be reduced to a two degrees of freedom problem, by referring the orbits to the Laplace plane. The main concern in this work was the position and stability of the equilibria in this problem, and in particular the generation of stable Kozai equilibria through bifurcation from a central equilibrium, which itself becomes unstable at high mutual inclination. This implies that, around the stability islands of the Kozai resonance, chaotic motion of the planets occurs.

Some articles have noted the importance of the Kozai resonance on the long-term behavior of extrasolar systems, when the orbital planes of the planets have a high mutual inclination (for instance, the case of the $v$ Andromedae system, Stepinski et al. 2000; Chiang et al. 2001). The Kozai resonance offers a secular phase-protection mechanism such that the system remains stable, even though both orbits may suffer large-scale variations both in eccentricity and inclination; in other words, the variations can be large but occur in a coherent fashion, dictated by the resonance condition, such that close approaches do not occur (see the original paper of Kozai 1962). Generally the goal of previous numerical studies was to analyze the stability of the detected systems, with regards to different mutual inclination values. The aim of the present work is different, as we try to examine the possibility of two-planet exosystems being locked in a stable Kozai-resonant state. In particular, a parametric study is undertaken for five real multi-planet systems that 
are not in mean-motion resonance ( $v$ Andromedae, HD 12661, HD 169830, HD 74156, HD 155358) by varying the (unknown) inclinations of their orbital planes and their nodal longitudes, thus changing their masses and mutual inclination.

The analytical works of Michtchenko et al. (2006) and Libert $\&$ Henrard $(2007,2008)$ on the Kozai resonance were developed in the Laplace-plane reference frame. In this frame, the Kozairesonant regions are characterized by the coupled variation of the eccentricity and inclination of the inner planet and the libration of its pericenter argument about $\pm 90^{\circ}$ (Kozai 1962). In this work we will show that, when a general reference frame is considered, the libration of this angle is not necessary for the system to be in Kozai resonance, so that a new characterization method is needed.

The paper is organized as follows. In Sect. 2, the Hamiltonian formulation of the 3D secular three-body problem of Libert \& Henrard (2007) is recalled, and a new characterization of the Kozai resonance is given for systems considered in a general reference frame. The possibility of extrasolar systems being in Kozai resonance is examined in Sect. 3. Our results are summarized in Sect. 4 and physical mechanisms that could generate the large mutual inclinations required for the Kozairesonant state are discussed.

\section{Hamiltonian formulation of the 3D secular three-body problem}

\subsection{Analytical modeling of the problem}

In this section we recall the analytical modeling of the 3D secular three-body problem and the main results obtained by Libert \& Henrard (2007). Let us consider a system consisting of a central star of mass $m_{0}$ and of two planets of mass $m_{1}$ and $m_{2}$, with $m_{1}$ being the closest to the central star. The Hamiltonian of this system, in the usual Jacobi coordinates, is (see e.g. Brouwer \& Clemence 1961):

$\mathcal{H}=-\frac{G m_{0} m_{1}}{2 a_{1}}-\frac{G m_{0} m_{2}}{2 a_{2}}-G m_{1} m_{2}\left[\frac{1}{\left|\boldsymbol{r}_{1}-\boldsymbol{r}_{2}\right|}-\frac{\left(\boldsymbol{r}_{1} \mid \boldsymbol{r}_{2}\right)}{r_{2}^{3}}\right]$,

where $a_{i}, \boldsymbol{r}_{i}, r_{i}$ and $G$ are, respectively, the osculating semi-major axis, the position vector, the norm of the position vector of the mass $m_{i}$, and the universal gravitational constant.

A set of canonical variables is formed by use of the classical modified Delaunay's elements (see for instance Laskar 1990):

$\lambda_{i}=$ mean longitude, $\quad L_{i}=m_{i} \sqrt{G m_{0} a_{i}}$

$p_{i}=-$ longitude of the pericenter,

$q_{i}=-$ longitude of the node,

$$
P_{i}=L_{i}\left[1-\sqrt{1-e_{i}^{2}}\right]
$$

$$
Q_{i}=L_{i} \sqrt{1-e_{i}^{2}}\left[1-\cos i_{i}\right]
$$

where $e_{i}$ and $i_{i}$ are the eccentricities and inclinations of the planets. In order to expand the last term of Eq. (1) in powers of the elements (i.e. a generalization of the Laplace-Lagrange secular theory to higher order in eccentricity and inclination, see e.g. Murray \& Dermott 1999), Libert \& Henrard (2007) used the non-dimensional variables $E_{i}=\sqrt{2 P_{i} / L_{i}}$ instead of the eccentricities $e_{i}$ and $S_{i}=\sqrt{2 Q_{i} / L_{i}}$ instead of the inclinations $i_{i}$; these variables are related to Delaunay's canonical variables and, at least for small to moderate eccentricities and inclinations, they have similar meanings and values.
For systems that are far from a mean motion resonance, the Hamiltonian of the system can be averaged over the "fast variables" $\lambda_{i}$ (to first order in the mass ratio), as in Libert \& Henrard (2007), to obtain the following expression:

$\mathcal{K}=\sum_{k, j_{1}, j_{2}, i_{l}, l \in \underline{4}} B_{i_{l}}^{k, j_{1}, j_{2}} E_{1}^{\left|j_{1}\right|+2 i_{1}} E_{2}^{\left|j_{2}\right|+2 i_{2}} S_{1}^{\left|k+j_{1}\right|+2 i_{3}} S_{2}^{\left|k+j_{2}\right|+2 i_{4}} \cos \Phi$,

where $\Phi=j_{1}\left(p_{1}-q_{1}\right)-j_{2}\left(p_{2}-q_{2}\right)-k\left(q_{1}-q_{2}\right)$. All variables $a_{i}$, $E_{i}, S_{i}, p_{i}$ and $q_{i}$ now designate values averaged over the fast variables $\lambda_{i}$. As the mean longitudes are ignorable, the associated moments $L_{i}$ are constant and so are the semi-major axes $a_{i}$. Then, this secular Hamiltonian describes a four degrees of freedom problem. Libert \& Henrard $(2007,2008)$ have shown that an expansion made of all terms such that the sum of the exponents in $E_{1}, E_{2}, S_{1}$ and $S_{2}$ is less or equal to 12 depicts the behavior of the system with enough accuracy, even when the eccentricities are as large as in many of the observed extrasolar systems.

In the three-body problem, Jacobi's reduction, also known as the elimination of the nodes (Jacobi 1842), allows us to reduce the Hamiltonian function (3) to a two degrees of freedom function only. The reduction is based on the existence of additional integrals of motion, namely the invariance of the total angular momentum, $\boldsymbol{C}$, both in norm and in direction. The constant direction of the vector $\boldsymbol{C}$ defines an invariant plane perpendicular to it. This plane is known as the invariant Laplace plane. The choice of this plane as plane of reference implies the following relations:

$q_{1}-q_{2}= \pm 180^{\circ}$

$\left(L_{1}-P_{1}\right) \cos i_{1}+\left(L_{2}-P_{2}\right) \cos i_{2}=C$

$\left(L_{1}-P_{1}\right) \sin i_{1}+\left(L_{2}-P_{2}\right) \sin i_{2}=0$

with $C$ being the norm of the total angular momentum. Another quantity, related to the total angular momentum, is frequently used. This is known as the angular momentum deficit (Laskar 1997):

$\mathrm{AMD}=\sum_{i=1}^{2} L_{i}\left(1-\sqrt{1-e_{i}^{2}} \cos i_{i}\right)=L_{1}+L_{2}-C$.

The dimensionless angular momentum deficit, denoted by the symbol $\Sigma$, has the following expression:

$\Sigma=\frac{A M D}{L_{2}}(1-\mu)$

where $\mu=m_{1} /\left(m_{1}+m_{2}\right)$. For a fixed value of this quantity (or equivalently for a fixed value of $C$ ), the relations (5)-(6) allow us to calculate the values of the inclinations as functions of the eccentricities.

As mentioned above, the problem is now reduced to two degrees of freedom only, described by the following Hamiltonian - see Libert \& Henrard (2007) for more details:

$\mathcal{K}=\sum_{n_{l}, l \in \underline{5}} E_{n_{l}} x_{1}^{n_{1}} y_{1}^{n_{2}} x_{2}^{n_{3}} y_{2}^{n_{4}} \chi^{n_{5}}$,

where $x_{i}, y_{i}$ are the new Poincaré-like canonical variables $\left(w_{i}\right.$ denotes the opposite of the (averaged) argument of the pericenter $\left.p_{i}-q_{i}\right)$, i.e.

$x_{i}=\sqrt{2 P_{i}} \cos w_{i}$ and $y_{i}=\sqrt{2 P_{i}} \sin w_{i}$

and $\chi$ is the part of the angular momentum deficit related to the mutual inclination of the orbits, i.e.

$\chi=\mathrm{AMD}-P_{1}-P_{2}=\mathrm{AMD}-\left(x_{1}^{2}+y_{1}^{2}+x_{2}^{2}+y_{2}^{2}\right) / 2$. 
Table 1. Parameters of the five exosystems analyzed in this work. All the parameters come from Butler et al. (2006), excepted those of the system HD 155358 from Schneider's catalog (http: //exoplanet. eu/).

\begin{tabular}{lccccccc}
\hline \hline & $a$ & $e$ & $\omega(\mathrm{deg})$ & $m \sin i\left(M_{\text {Jup }}\right)$ & $M_{\text {Star }}\left(M_{\text {Sun }}\right)$ & error on $e$ & error on $\omega(\mathrm{deg})$ \\
\hline$v$ And & 0.832 & 0.262 & 245.5 & 1.98 & 1.32 & 0.021 & 5.3 \\
(c-d) & 2.54 & 0.258 & 279 & 3.95 & & 0.032 & 10 \\
HD 12661 & 0.831 & 0.361 & 296.3 & 2.34 & 1.11 & 0.011 & 2.6 \\
& 2.86 & 0.017 & 38 & 1.83 & & 0.029 & 0 \\
HD 169830 & 0.817 & 0.310 & 148 & 2.9 & 1.43 & & \\
& 3.62 & 0.33 & 252 & 4.1 & & & \\
HD 74156 & 0.29 & 0.6360 & 181.5 & 1.8 & 1.21 & & \\
& 3.35 & 0.583 & 242.4 & 6 & & & \\
HD 155358 & 0.628 & 0.112 & 162 & 0.89 & 0.87 & & \\
& 1.224 & 0.176 & 279 & 0.504 & & & \\
\hline
\end{tabular}

Thus, the evolution of the system can be followed on the $(x, y)$ plot, where, at any given moment, the polar radius is roughly proportional to the eccentricity of the planet and the polar angle is the argument of pericenter. Note that the coefficients $E_{n_{l}}$ only depend on the mass ratio and the ratio of the semi-major axes.

\subsection{Characteristics of the Kozai resonance in a general reference frame}

According to Libert \& Henrard (2007), the origin $x_{1}=x_{2}=y_{1}=$ $y_{2}=0$ is an equilibrium of the averaged planetary three-body problem, reduced to two degrees of freedom. This equilibrium corresponds to circular orbits, and, according to (7), to the maximal mutual inclination compatible with a fixed value of AMD. When the angular momentum deficit is small, this equilibrium is stable. For larger mutual inclinations, it becomes unstable, generating a large chaotic zone and, by bifurcation, two regular regions, the so-called Kozai resonances (Michtchenko et al. 2006). These resonant regions are characterized, in the Laplaceplane reference frame that we are using, by the coupled variation of the eccentricity and the inclination of the inner planet and the libration of the argument of the pericenter of the same planet $\omega_{1}$ around $\pm 90^{\circ}$ (Kozai 1962). The critical value of the mutual inclination, which corresponds to the change of stability of the central equilibrium, has been calculated by Jefferys et al. (1966) and Robutel (1995), for vanishingly small semi-major axis ratios and by Libert \& Henrard (2007) for values of the semi-major axes and mass ratios compatible with those of extrasolar systems. For mass ratios between 0.5 and 2 , the critical mutual inclination is of the order of $40^{\circ}-45^{\circ}$.

The aforementioned definition of the Kozai resonance is related to the use of the Laplace-plane reference frame. We can ask ourselves whether this characterization is also valid for a general reference frame, such as the one formed e.g. by considering the plane of the sky (perpendicular to the line of sight) as our reference plane. In the following, we will adopt this general reference frame and hence the angle $i_{i}$ will denote the inclination of the orbital plane of the mass $m_{i}$ with respect to the plane of the sky.

The libration of the argument of the pericenter of the inner planet $\omega_{1}$ around $\pm 90^{\circ}$ is not a necessary characteristic of the Kozai resonance, when the system is viewed in a general reference frame. To show this, we use the HD 169830 system (see Table 1), assuming initial inclinations of $45^{\circ}$ for both orbital planes (i.e. masses multiplied by $2 / \sqrt{2}$ ) and $\Omega_{1}=81.5^{\circ}$, $\Omega_{2}=0^{\circ}$, i.e. a mutual inclination value of $I_{\text {mut }}=55^{\circ}$ (see Eq. (12) below). Figure 1 represents the time evolution of the orbital elements of this system (on the left) and the same system referred to the Laplace-plane reference frame (on the right). To translate into the Laplace plane, we let the planets evolve until their nodal lines are aligned. Once the system reaches this configuration, we switch to the Laplace plane by using the relations (5) and (6). In our example, we obtain: $i_{1}^{\prime}=31.79^{\circ}$, $i_{2}^{\prime}=9.30^{\circ}, \omega_{1}^{\prime}=273.4^{\circ}, \omega_{2}^{\prime}=230.8^{\circ}, \Omega_{1}^{\prime}=180^{\circ}$ and $\Omega_{2}^{\prime}=0^{\circ}$, where the prime symbol refers to the Laplace-plane reference frame. As shown in Fig. 1, the system is well in Kozai resonance since, in the Laplace plane, we observe a coupled variation of the eccentricity and inclination of the planet $m_{1}$ and the libration of $\omega_{1}^{\prime}$ around $270^{\circ}$. However, in the general reference frame, the argument of the pericenter $\omega_{1}$ circulates, even though the system is in Kozai resonance.

The coupling between the eccentricity and inclination of the inner planet is also a feature that is not observed in both reference frames. Indeed, no obvious relation between the elements $e_{1}$ and $i_{1}$ of the system can be deduced in the general reference frame, just by looking at the long-term behavior in Fig. 1.

To better understand the characteristics of the Kozai resonance and the coupling between the orbital elements that it induces, independent of the adopted reference frame, we propose here a new method in which we identify and order (by amplitude) the main frequencies in the timeseries of each orbital element to reveal the existing correlations.

Before analyzing the case of a system in Kozai resonance, let us recall the main results of Libert \& Henrard (2008) about the decomposition in frequencies of a system with a secular, non-resonant, behavior. First we focus on the HD 12661 system (see parameters in Table 1), assuming an inclination of $i=30^{\circ}$ for both orbital planes (i.e. double masses) and a mutual inclination of $30^{\circ}$. The orbital evolution of this system is represented in Fig. 2, both in the general reference frame and in the Laplace-plane frame. In this example we adopt the maximal value of the argument $\omega_{1}$ that is consistent with the observational data (i.e. $\omega_{1}=298.9^{\circ}$ ). We perform frequency analysis on the corresponding timeseries, using the algorithm first introduced by Champenois (1998), based on the original work of Laskar (1993).

Table 2 (left panel) summarizes the main spectral lines that dominate the long-term behavior of the orbital elements defined in the general reference frame. The trigonometric terms are listed in descending amplitude, starting from $c_{1}$ (largest amplitude) down to $c_{4}$. Bold type $c_{1}$ denotes the mean precession rate of an angular variable in circulation. As suggested by Fig. 2 (left), the eccentricities $e_{1}$ and $e_{2}$ have the same spectral decomposition. The same is true for the inclinations $i_{1}$ and $i_{2}$. Furthermore, we remark that the decomposition of $e_{i}$ is strongly related to the one of $\Delta \varpi=\varpi_{1}-\varpi_{2}$. The main frequency of the two eccentricities equals the mean precession rate of the difference of the 

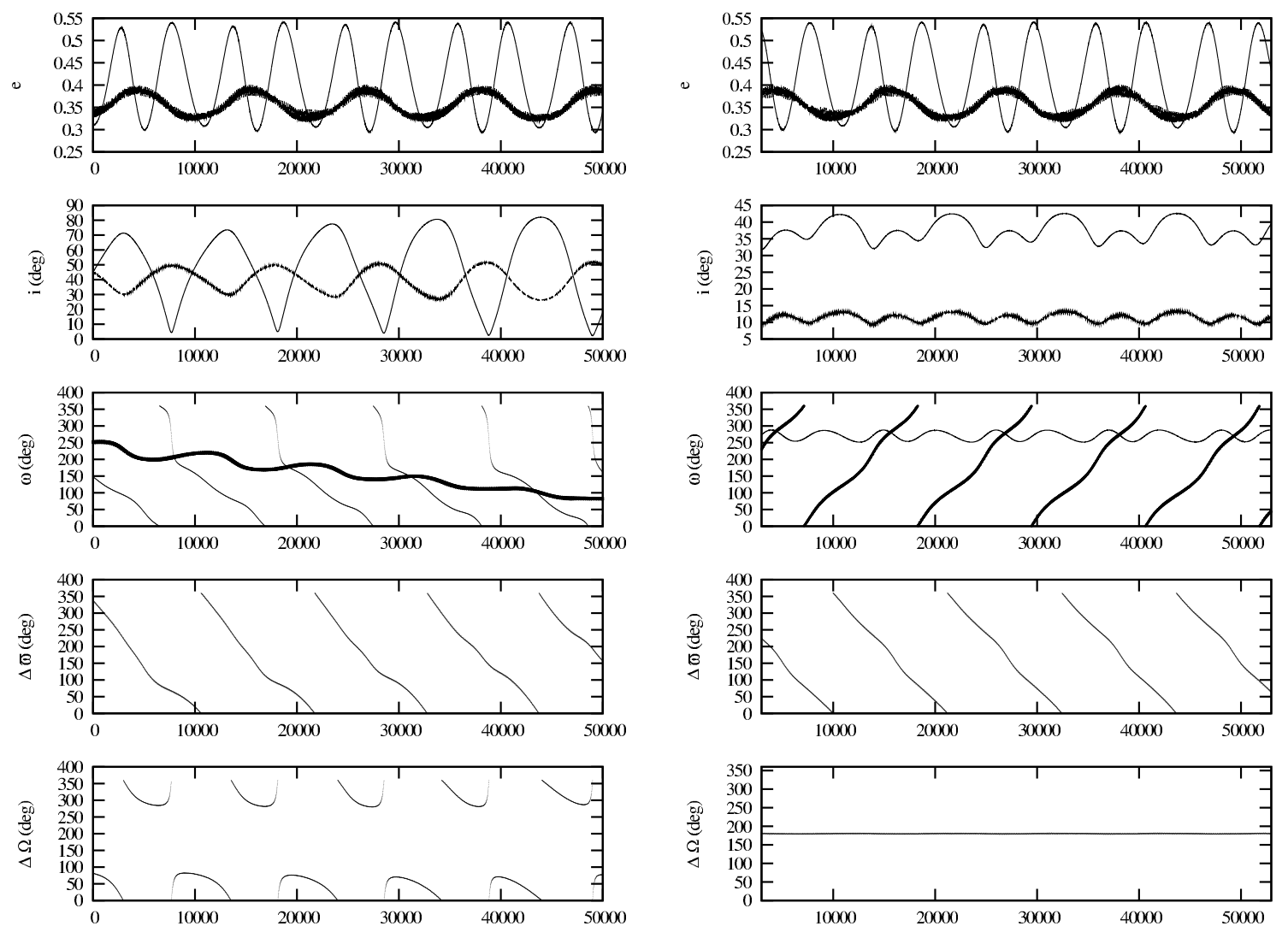

Fig. 1. Time variations on $5 \times 10^{5}$ years of the exosystem HD 169830 with $i_{1}=i_{2}=45^{\circ}$ and $I_{\text {mut }}=55^{\circ}$ in the general sky plane reference frame (on the left) and in the Laplace-plane reference frame (on the right). The dotted lines stand for the outer body $m_{2}$. The integrations are performed with Swift (with the time of periastron passage of the outer planet as the initial epoch). Even though the system is in Kozai resonance, the argument $\omega_{1}$ circulates in the general reference frame.
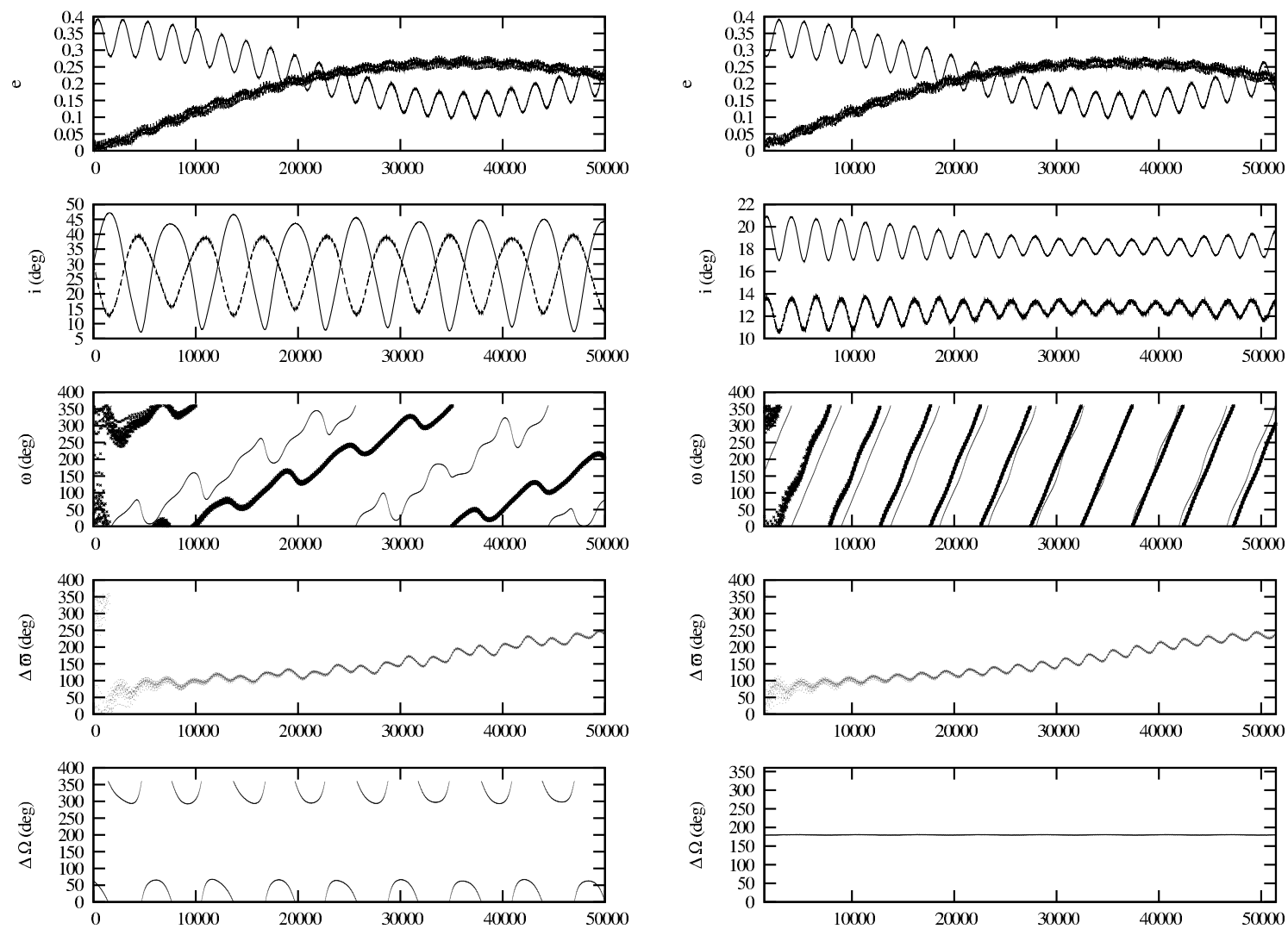

Fig. 2. Long-term time evolution of the exosystem HD 12661 with $i_{1}=i_{2}=30^{\circ}$ and $I_{\text {mut }}=30^{\circ}$ in the general sky plane reference frame (on the left) and in the Laplace-plane reference frame (on the right). The dotted lines stand for the outer body $m_{2}$. 
Table 2. Main results concerning the long-term behavior of the system HD 12661 with $i_{1}=i_{2}=30^{\circ}$ and a mutual inclination of $30^{\circ}$, obtained by decompositions in frequencies of the elements reproduced in Fig. 2. The system is analyzed in the general sky plane reference frame (left table) and in the Laplace-plane reference frame (right table). The periods are expressed in years.

\begin{tabular}{rlllllll}
\hline \hline Periods & $e$ & $i$ & $\omega_{1}$ & $\omega_{2}$ & $\Delta \varpi$ & $\Delta \Omega$ & $2 \omega_{1}$ \\
\hline 70508 & $c_{1}$ & & & $c_{2}$ & $\mathbf{c}_{1}, c_{2}$ & & \\
2380 & $c_{2}$ & & & & $c_{4}$ & & \\
35254 & $c_{3}$ & & & & & & \\
2464 & $c_{4}$ & & & & & $c_{4}$ & \\
6059 & & $c_{1}$ & $c_{2}$ & & & $c_{1}$ & $c_{2}$ \\
3030 & & $c_{2}$ & & & & & \\
3921 & & $c_{3}$ & & & & & \\
2020 & & $c_{4}$ & & & & $c_{2}$ & \\
22218 & & & $\mathbf{c}_{1}$ & & & & \\
3166 & & & $c_{3}$ & & & & $c_{4}$ \\
6629 & & & $c_{4}$ & & & & \\
32439 & & & & $\mathbf{c}_{1}$ & & & \\
5580 & & & & $c_{3}$ & & & \\
5171 & & & & $c_{4}$ & & & \\
2303 & & & & & $c_{3}$ & & \\
1211 & & & & & & & \\
11109 & & & & & & & \\
3166 & & & & & & &
\end{tabular}

longitudes of the pericenters. The same coupling between the two eccentricities is found in the second-order LaplaceLagrange secular theory (see e.g. Murray \& Dermott (1999)). Thus, neglecting short-period terms, the extrema of $e_{i}$ are reached when $\sin \Delta \varpi=0$ and, for similar reasons, the extrema of $i_{i}$ when $\sin \Delta \Omega=0$.

The same system can be viewed and analyzed in the Laplaceplane frame. As shown by Fig. 2 (right), the behavior of the inclinations and of the angles is quite different, in terms of frequencies (for a quantitative measure, see Table 2, right panel). However, once again, both eccentricities are influenced by the same frequencies and in the same order as before; the inclinations $i_{1}^{\prime}$ and $i_{2}^{\prime}$ also have the same decomposition, but with very different frequencies than $i_{1}$ and $i_{2}$. Thus the choice of the reference frame does not influence the coupling of the eccentricities, but changes the coupling between the elements such that the local extrema of $e_{i}^{\prime}$ and $i_{i}^{\prime}$ are reached when $\sin 2 \omega_{1}^{\prime}=0$ and the global extrema of $e_{i}^{\prime}$ and $i_{i}^{\prime}$ when $\sin \Delta \varpi^{\prime}=0$.

In order to perform the same analysis, but for a system in Kozai resonance, let us consider the same exosystem HD 12661 but assume now a higher value for the mutual inclination $\left(45^{\circ}\right)$. As shown by the long-term behavior, represented in Fig. 3, such a system is well in a Kozai-resonant state. A quick look at the figures enables us to see a major difference with respect to the previous case: the eccentricities are not influenced by the same frequencies any longer.

When studying the system in the general reference frame, the frequency analysis (summarized in Table 3, left panel) reveals the following couplings (neglecting short-period terms): the inclinations have the same frequency decomposition and reach their extrema when $\sin \Delta \Omega=0$, while the eccentricity of the outer planet reaches its extrema when $\sin \Delta \varpi=0$. The two main frequencies of $\omega_{1}$ are such that the main one coincides with the main (common) frequency of $i_{1}$ and $i_{2}$, while the second one is the same as the main frequency of the eccentricity of the inner planet, $e_{1}$. The same kind of behavior is observed in all cases that we found to be in Kozai resonance (as verified by moving to the Laplace-plane reference frame); namely, the main frequency of the planetary inclinations is also the main frequency of $\omega_{1}$, while the fundamental mode of $e_{1}$ is not the main frequency of $\Delta \varpi$. As a result, the eccentricities of both planets are not coupled.

We also perform a frequency analysis on the timeseries of the orbital elements of the same system, as defined in the Laplaceplane frame. As expected, we found the features described in the work of Kozai (1962). The reduction to the Laplace plane simplifies the relations between the orbital elements, since there are now two main frequencies instead of three: again neglecting short-period terms, the eccentricity of $m_{1}$ as well as the inclinations of both planets reach extremal values when $\sin 2 \omega_{1}^{\prime}=$ 0 while the eccentricity of the planet $m_{2}$ is extremal when $\sin \Delta \varpi^{\prime}=0$. Thus, the eccentricities $e_{1}^{\prime}$ and $e_{2}^{\prime}$ are again uncoupled, but $e_{1}^{\prime}, i_{1}^{\prime}, i_{2}^{\prime}$ and $\omega_{1}^{\prime}$ now have a common fundamental frequency: the frequency of libration of the resonant angle.

In conclusion, we have compared in this section the dynamical behavior of a system in Kozai resonance to a secular, nonresonant, system. We conclude that the main characteristic that can be used to descriminate between the two cases is the coupling or non-coupling of the eccentricities of the two planets. Furthermore, we have shown that the libration of the argument of the pericenter of the inner planet and the coupling between its eccentricity and inclination (i.e. the "usual" definition of the Kozai resonance) depends upon adopting the Laplace-plane reference frame. On the other hand, the Kozai resonance can safely be identified in an abstract reference frame, using frequency analysis; The system is in Kozai resonance if $e_{1}$ and $e_{2}$ are uncoupled and the main frequency of $\omega_{1}$ is the fundamental mode of the inclinations. We will use this criterion to identify the Kozai resonance, in the parametric study of the five systems that follows.

\subsection{Illustration}

To visualize the different dynamics between a system with the orbital parameters of HD 12661 and $I_{\text {mut }}=30^{\circ}$ or $45^{\circ}\left(i_{1}=\right.$ $i_{2}=30^{\circ}$ as in the previous section), we can draw for both cases the so-called representative plane, introduced by Libert \& Henrard (2007). This is the plane for which $x_{1}=x_{2}=0$, i.e. both pericenter arguments are fixed to $\pm 90^{\circ}$. We insist on the fact that this plane is not a surface of section, as the phase-space is four dimensional. However, almost all orbits have to cross the 

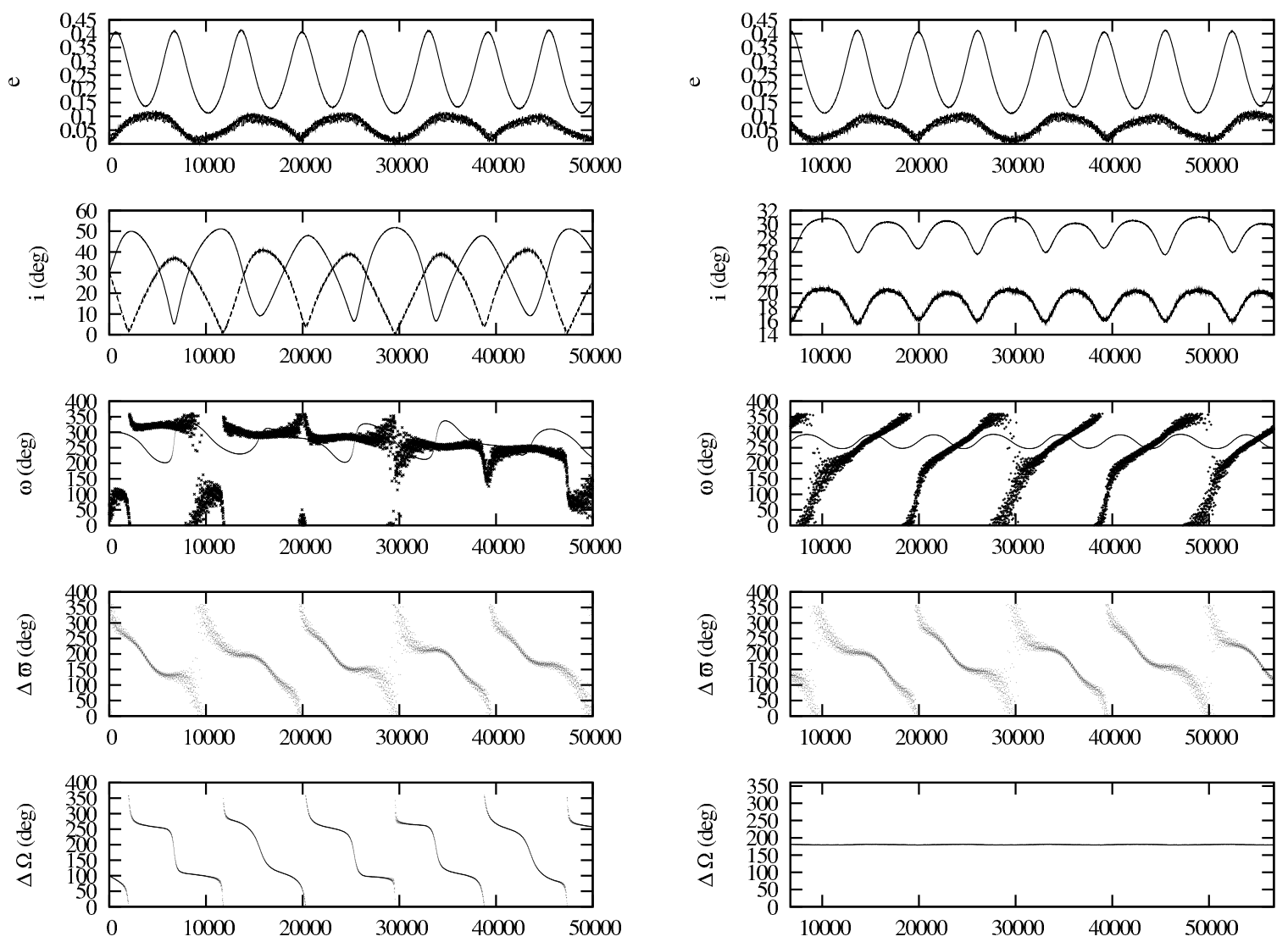

Fig. 3. Same representation as Fig. 2 for the same system considered with a higher mutual inclination: $I_{\text {mut }}=45^{\circ}$.

Table 3. Main results concerning the long-term behavior of the system HD 12661 with $i_{1}=i_{2}=30^{\circ}$ and a mutual inclination of $45^{\circ}$, obtained by decompositions in frequencies of the elements reproduced in Fig. 3. The system is analyzed in the general sky plane reference frame (left table) and in the Laplace-plane reference frame (right table). The periods are expressed in years.

\begin{tabular}{|c|c|c|c|c|c|c|c|c|}
\hline Periods & $\overline{e_{1}}$ & $\overline{e_{2}}$ & $\bar{i}$ & $\overline{\omega_{1}}$ & $\overline{\overline{\omega_{2}}}$ & $\overline{\Delta \varpi}$ & $\overline{\Delta \Delta \Omega}$ & $2 \omega_{1}$ \\
\hline 6439 & $c_{1}$ & & & $c_{2}$ & & & & $c_{2}$ \\
\hline 9980 & $c_{2}$ & $c_{1}$ & & & $c_{4}$ & $-\mathbf{c}_{1}, c_{2}$ & & \\
\hline 3220 & $c_{3}$ & & & & & & & \\
\hline 18152 & $c_{4}$ & $c_{2}$ & & & & $c_{4}$ & & \\
\hline 4904 & & $c_{3}$ & & & & & & \\
\hline 3914 & & $c_{4}$ & & & & $c_{3}$ & & \\
\hline 9092 & & & $c_{1}$ & $c_{1}$ & $c_{2}$ & & $-\mathbf{c}_{1}, c_{4}$ & $c_{1}$ \\
\hline 4546 & & & $c_{2}$ & $c_{3}$ & & & $c_{2}$ & \\
\hline 22069 & & & $c_{3}$ & & & & & $c_{3}$ \\
\hline 3770 & & & $c_{4}$ & $c_{4}$ & & & & $c_{4}$ \\
\hline 3031 & & & & & & & $c_{3}$ & \\
\hline 102261 & & & & & $-\mathbf{c}_{1}, c_{3}$ & & & \\
\hline
\end{tabular}

representative plane, and the points of intersection have to follow a constant energy curve. For a better picture of the dynamics, we plot several level curves of (9) on the plane $\left(e_{1}^{\prime} \sin w_{1}^{\prime}, e_{2}^{\prime} \sin w_{2}^{\prime}\right)$ for $w_{i}^{\prime}= \pm 90^{\circ}$. Figure 4 shows the representative planes obtained for the parameters of the HD 12661 system with $I_{\text {mut }}=30^{\circ}$ (left panel) and $I_{\text {mut }}=45^{\circ}$ (right panel). In the first case, the equilibrium is stable and corresponds to circular orbits. In the second case, as the mutual inclination is higher than the critical value, the equilibrium is unstable and gives birth by bifurcation to the two (stable) Kozai equilibria.

The crosses represent the intersections of the two "HD 12661" orbits with the representative planes. For the lower value of $I_{\text {mut }}$, the four intersection points correspond to $\left(e_{1}^{\prime} \mathrm{min}\right.$, $\left.e_{2}^{\prime} \max \right)$ when $\left(\omega_{1}^{\prime}, \omega_{2}^{\prime}\right)=\left(90^{\circ}, 90^{\circ}\right),\left(270^{\circ}, 270^{\circ}\right)$ and $\left(e_{1}^{\prime} \max\right.$, $\left.e_{2}^{\prime} \min \right)$ when $\left(\omega_{1}^{\prime}, \omega_{2}^{\prime}\right)=\left(90^{\circ}, 270^{\circ}\right),\left(270^{\circ}, 90^{\circ}\right)$. The fact that each intersection point is located in a different quadrant of the plane shows that the angle $\omega_{1}^{\prime}\left(\right.$ and $\left.\omega_{2}^{\prime}\right)$ circulates.

On the contrary, all intersection points in the right panel of Fig. $4\left(\left(e_{1}^{\prime} \min , e_{2}^{\prime} \min \right)\right.$ for $\left(\omega_{1}^{\prime}, \omega_{2}^{\prime}\right)=\left(270^{\circ}, 90^{\circ}\right),\left(e_{1}^{\prime} \min\right.$, $\left.e_{2}^{\prime} \max \right)$ for $\left(270^{\circ}, 270^{\circ}\right),\left(e_{1}^{\prime} \max , e_{2}^{\prime} \min \right)$ for $\left(270^{\circ}, 90^{\circ}\right)$ and $\left(e_{1}^{\prime} \max , e_{2}^{\prime} \max \right)$ for $\left.\left(270^{\circ}, 270^{\circ}\right)\right)$ are situated on the right-hand side of the representative plane, which means that the argument of pericenter of the inner planet $\omega_{1}^{\prime}$ librates around $270^{\circ}$. Hence, it is obvious from Fig. 4 that the HD 12661 system must be in Kozai resonance, if $I_{\text {mut }}=45^{\circ}$. It is important to note the fact that, even if the system seems to be located close to what looks like a "separatrix", no information regarding the stability of the system can be deduced from this representation alone, as it is neither a surface of section nor a complete plot of the orbits. 

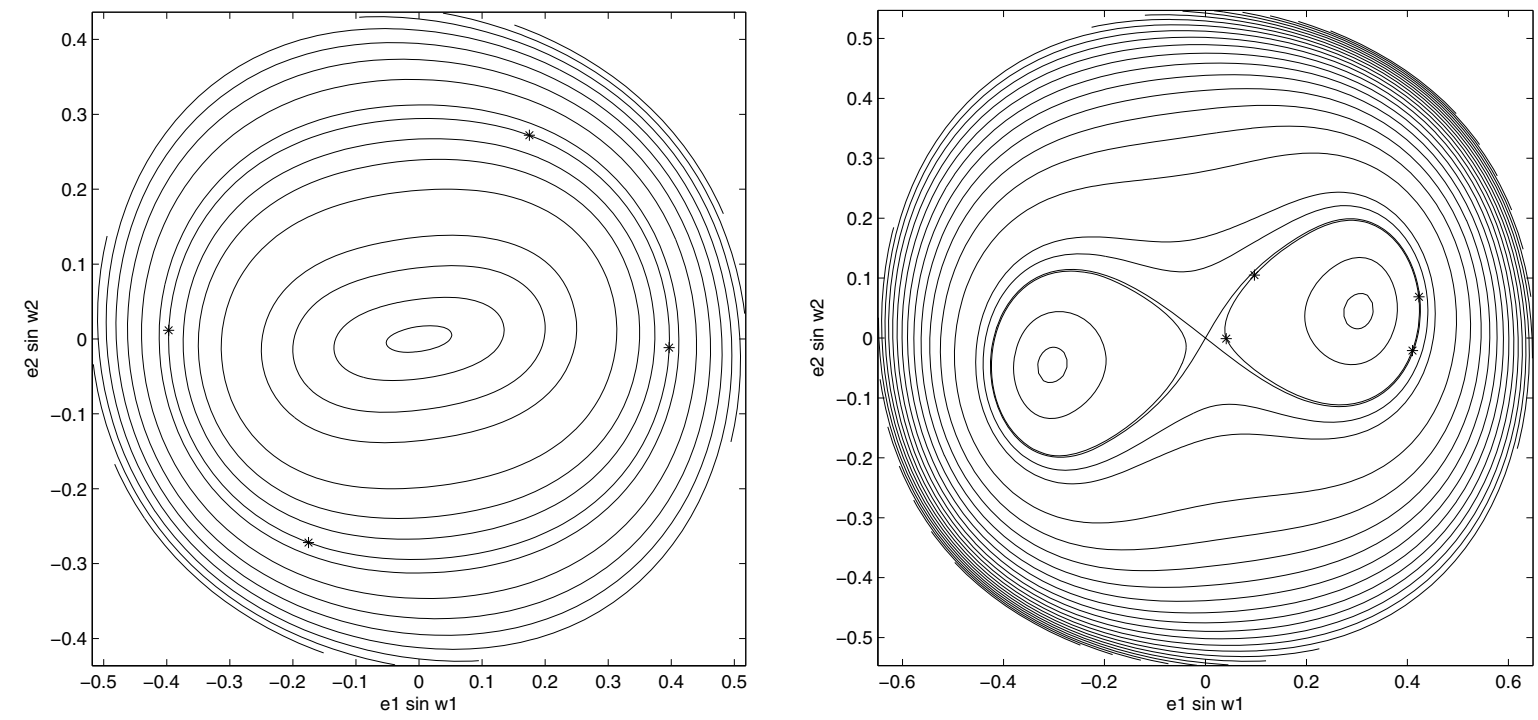

Fig. 4. Representative planes $\left(e_{1}^{\prime} \sin w_{1}^{\prime}, e_{2}^{\prime} \sin w_{2}^{\prime}\right)$ for the HD 12661 system with $I_{\text {mut }}=30^{\circ}\left(a_{1} / a_{2}=0.294, m_{1} / m_{2}=1.279, \Sigma=0.0440\right)$ on the left and with $I_{\mathrm{mut}}=45^{\circ}\left(a_{1} / a_{2}=0.284, m_{1} / m_{2}=1.279, \Sigma=0.0714\right)$ on the right. The difference in the dynamics of the two cases is obvious.

\section{Possible extrasolar systems in Kozai resonance}

In this section we probe the possibility that some of the observed extrasolar systems can harbor Kozai-type motion. We remind the reader that observational uncertainties and/or incomplete modeling from our part (e.g. the absence of general-relativistic precession) are such that one cannot identify candidate systems for Kozai-type motion with the desired certainty; this is not the purpose of this study. What we wish to show is that a large fraction of the observed multi-planet systems has physical (masses) and orbital (orbital separations, eccentricities and apsidal-line orientations) parameters that are consistent with 3D Kozai-type motion, provided that the mutual inclination is above a critical value.

\subsection{Methodology}

At present, 25 multiple extrasolar systems have been detected. Among them, we analyze five multi-planet systems, which were selected according to the following criteria: (a) they have two significant planets that are not in mean-motion resonance; (b) these planets have masses less than $10 M_{\text {Jup }}$ and (c) their orbital periods are longer than 30 days. We note that $v$ Andromedae is a three-planet system, but the innermost planet, $v$ And $\mathrm{b}$, has a smaller mass than the other two, is very close to the $\operatorname{star}(a \approx 0.06 \mathrm{AU})$ and has a very small eccentricity $(e \approx 0.03)$, so that its effect on the other two planets is small enough to ignore, to a first approximation (Stepinski et al. 2000). Similarly, a third planet candidate recently has been announced for HD 74156 (Bean et al. 2008). This is a Saturn-sized planet but, interestingly enough, is supposed to orbit between the two larger, previously known, planets. Although Barnes et al. (2008) suggest that this planet was in fact "predicted" as a pre-requisite for the stability of the system, Baluev (2007) on the other hand argues that in fact it may be a false detection, caused by annual systematic errors. For these reasons we decided not to take into account this planet and proceed in analyzing HD 74156, assuming only the two previously established planets.

Table 1 gives the parameters of the five selected systems as well as the errors on the values of the eccentricities and arguments of pericenters for two of them. We adopt the parametrization of Butler (2006) for $v$ Andromedae, HD 12661, HD 169830 and HD 74156. The parameters of the exosystem HD 155358 can be found in the Extrasolar Planets Catalog of Jean Schneider, available on the web site http: //exoplanet. eu/catalog.php. Let us recall that, as the spatial resolution of their orbits is currently impossible, we have no information on the inclinations of their orbital planes to the plane of the sky ${ }^{1}(i)$ and on the longitudes of their nodes $(\Omega)$, but also we only have a poor determination of the masses: the quantities $m_{i} \sin i_{i}$ are the observed masses of the planets, corresponding to the real ones only in the case of $i=90^{\circ}$. Thus, these values constitute lower bounds to the real mass values.

To examine the possibility of the five systems of Table 1 being locked in a Kozai-resonant state, we compute the long-term orbital behavior of each system, assuming different values of the (unknown) inclinations of the orbital planes to the plane of the sky and of the (unknown) nodal longitudes (sky-plane reference frame). The numerical integrations are performed using the symplectic method of Wisdom \& Holman (1991), as is implemented in the SWIFT package (Levison \& Duncan 1994). Thus, this study neglects the post-Newtonian effects, mainly the generalrelativistic precession caused by the host star, which may give significant corrections for planets with very small orbital radii. We choose to vary both inclinations of the orbital planes in the same way $\left(i_{1}=i_{2}=i\right)$, to keep the mass ratio constant. To increase the mutual inclination of the two orbital planes, we vary the difference of the nodal longitudes since

$\cos I_{\mathrm{mut}}=\cos i_{1} \cos i_{2}+\sin i_{1} \sin i_{2} \cos \Delta \Omega$.

Note that, for a fixed value of $I_{\text {mut }}$, the two cases $\Delta \Omega\left(=\Omega_{1}-\right.$ $\left.\Omega_{2}\right)<0$ and $\Delta \Omega>0$ have to be treated separately as they do not correspond to the same configuration.

Five values of the inclination $i$ are considered $(i=$ $90^{\circ}, 75^{\circ}, 60^{\circ}, 45^{\circ}, 30^{\circ}$ ), which means that the considered masses of the planets are between one and two times the reported values. Concerning the values of the longitudes of the nodes, we select them in such a way that the mutual inclination, defined by Eq. (12), is equal to $40^{\circ}, 45^{\circ}, 50^{\circ}, 55^{\circ}$ and $60^{\circ}-$ in general higher

\footnotetext{
1 Harrington et al. (2006) have found a lower bound of $i=30^{\circ}$ for the planet $v$ And $\mathrm{b}$ that is not considered in this study.
} 
Table 4. Results of the numerical computation of the long-term behavior of the five systems assuming different values of the common inclination of the orbital plane to the plane of the sky $(i)$ and of the difference of the nodal longitudes $(\Delta \Omega)$. In this table are summarized the different occurrences of the Kozai-resonant state of the five systems with high mutual inclination and an indication of the percentage of chaos observed in the results.

\begin{tabular}{|c|c|c|c|c|}
\hline Exosystems & Inclination & $\begin{array}{l}I_{\text {mut }} \text { of the Kozai-resonant state } \\
\text { for } \Delta \Omega<0\end{array}$ & $\begin{array}{l}I_{\text {mut }} \text { of the Kozai-resonant state } \\
\text { for } \Delta \Omega>0\end{array}$ & $\%$ of chaoticity \\
\hline$v$ Andromedae & $i=30^{\circ}$ & $45-60$ (lib) & 60 (lib) & $\pm 80 \%$ \\
\hline HD 12661 & $i=45^{\circ}$ & - & $55-60$ & $\pm 50 \%$ \\
\hline & $i=30^{\circ}$ & 60 (lib) & $45-60$ (lib) & \\
\hline HD 169830 & $i=90^{\circ}$ & $55-60$ & $55-60$ & $\pm 40 \%$ \\
\hline & $\begin{aligned} i & =75^{\circ} \\
i & =60^{\circ} \\
i & =45^{\circ}\end{aligned}$ & $\begin{array}{l}- \\
- \\
-\end{array}$ & $\begin{array}{l}50-60 \\
50-60 \\
45-60\end{array}$ & \\
\hline HD 74156 & $\begin{aligned} i & =90^{\circ} \\
i & =75^{\circ} \\
i & =60^{\circ} \\
i & =45^{\circ}\end{aligned}$ & $\begin{array}{l}45-60 \\
45-60 \\
50-60 \\
45-60\end{array}$ & $\begin{array}{c}45-60 \\
45-60 \\
45-60 \\
-\end{array}$ & $\pm 30 \%$ \\
\hline HD 155358 & - & - & - & $\pm 80 \%$ \\
\hline
\end{tabular}

values than the critical mutual inclination. For each integration, all the other parameters are the same as in Table 1.

\subsection{Numerical results}

The results of the computation of the long-term behaviors of the five systems, analyzed according to the criterion introduced in Sect. 2.2, are summarized in Table 4. For each inclination, $i$, that is considered, the mutual inclination range, corresponding to a Kozai-resonant state, is given. The notation (lib) indicates a Kozai-resonant state that is also characterized by the libration of $\omega_{1}$ in the sky-plane reference frame. In the last column, we indicate, for each exosystem, the percentage of integrations that turned out to be chaotic, in the sense that we observed a nonperiodicity in the orbital evolution in less than 100000 years. This non-periodicity may be related to the chaos that develops around the stability islands of the Kozai resonance, as will be shown by the surfaces of section in Sect. 3.3.

According to Table 4, four of the five systems can be in a Kozai-resonant state, as their eccentricities and secular phases are such that the system would be placed inside the island of stable motion of the Kozai resonance, provided that their masses and mutual inclination are high enough. For the $v$ Andromedae system, only double the reported masses (i.e. $i=30^{\circ}$ ) would enable the system to be in Kozai resonance. Note that a high percentage of chaotic behavior is observed when we consider a high initial mutual inclination for this system. These results are in agreement with Fig. 8 of Stepinski (2000), which reports small variations of the eccentricities in the case of high mutual inclination and double masses of the planets. As we see, these small variations at high mutual inclination are due to the Kozai mechanism. The same kind of behavior is observed for the HD 12661 system, for which, however, many more cases turn out to be stable. Note that only for these two systems is the Kozai resonance also revealed by the libration of $\omega_{1}$ (in the sky-plane reference frame).

The systems HD 169830 and HD 74156 have a quite similar behavior, in the sense that the possibility of the Kozai resonance exists for a large range of masses and mutual inclinations. The planets of these two systems are far from each other (HD 74156 is even a hierarchical system ${ }^{2}$ ), which explains the small amount of chaotic evolution. Furthermore, both masses already being

\footnotetext{
${ }^{2}$ We remind the reader that we have chosen to ignore the HD $74156 \mathrm{~d}$ dubious planet candidate.
}

quite high, the Kozai resonance is only present for large values of the inclination $i$.

Finally, no Kozai behavior was found in any representation of the HD 155358 system. Only a few regular solutions were found. This is because of the relatively small orbital separation between the two planets.

\subsection{Sensitivity to observational uncertainties}

Uncertainties in the observational data are large. Table 1 gives an idea of the observational errors on the orbital parameters of the two "well-observed" systems, $v$ Andromedae and HD 12661. Even if these errors are quite small, we show in this section that they can have an important effect on the dynamical state of these systems.

To see the influence of the observational errors on the Kozairesonant states identified in the previous section, we perform a set of numerical integrations in which the best-fit parameter values of Table 1 are used for all initial elements except one (either $e_{1}, e_{2}, \omega_{1}$ or $\left.\omega_{2}\right)$. The extremal values for this element are always considered.

We find that the Kozai resonance is in general not influenced by these small changes, except when the initial mutual inclination is assumed close to the critical value. For instance, the systems $v$ Andromedae and HD 12661 (with $i=30^{\circ}$ ) are quite sensitive to the observational errors for $I_{\text {mut }} \approx 45^{\circ}$, but not for higher values. In this case, the most influential initial parameter seems to be the argument of pericenter of the inner planet, $\omega_{1}$.

In order to illustrate these results, we draw, in Fig. 5, two representative planes for the HD 12661 system $\left(i_{1}=i_{2}=30^{\circ}\right.$ and $I_{\text {mut }}=45^{\circ}$ ), assuming the minimum (left) or maximum (right) value for $\omega_{1}$. Note that the difference is less than $3^{\circ}$, but it induces a significant change in the dynamics. Indeed, in the first case, the argument of pericenter $\omega_{1}^{\prime}$ circulates (no Kozai), while in the second case it librates (Kozai).

This change of dynamics is more obvious on the surfaces of section, displayed in Fig. 6. We define the surface of section by setting $x_{2}=0$ (i.e. $w_{2}^{\prime}= \pm 90^{\circ}$ ) with $\dot{x}_{2}<0$, projected on the plane $\left(e_{1}^{\prime} \cos w_{1}^{\prime}, e_{2}^{\prime} \sin w_{2}^{\prime}\right)$. We refer to Libert \& Henrard (2007) for more details. For the maximum value of $\omega_{1}$ (right), the motion is regular. On the contrary, for the minimum value of $\omega_{1}$ (left), chaotic motion is observed; the orbit follows the unstable manifolds emanating from the central equilibrium. 

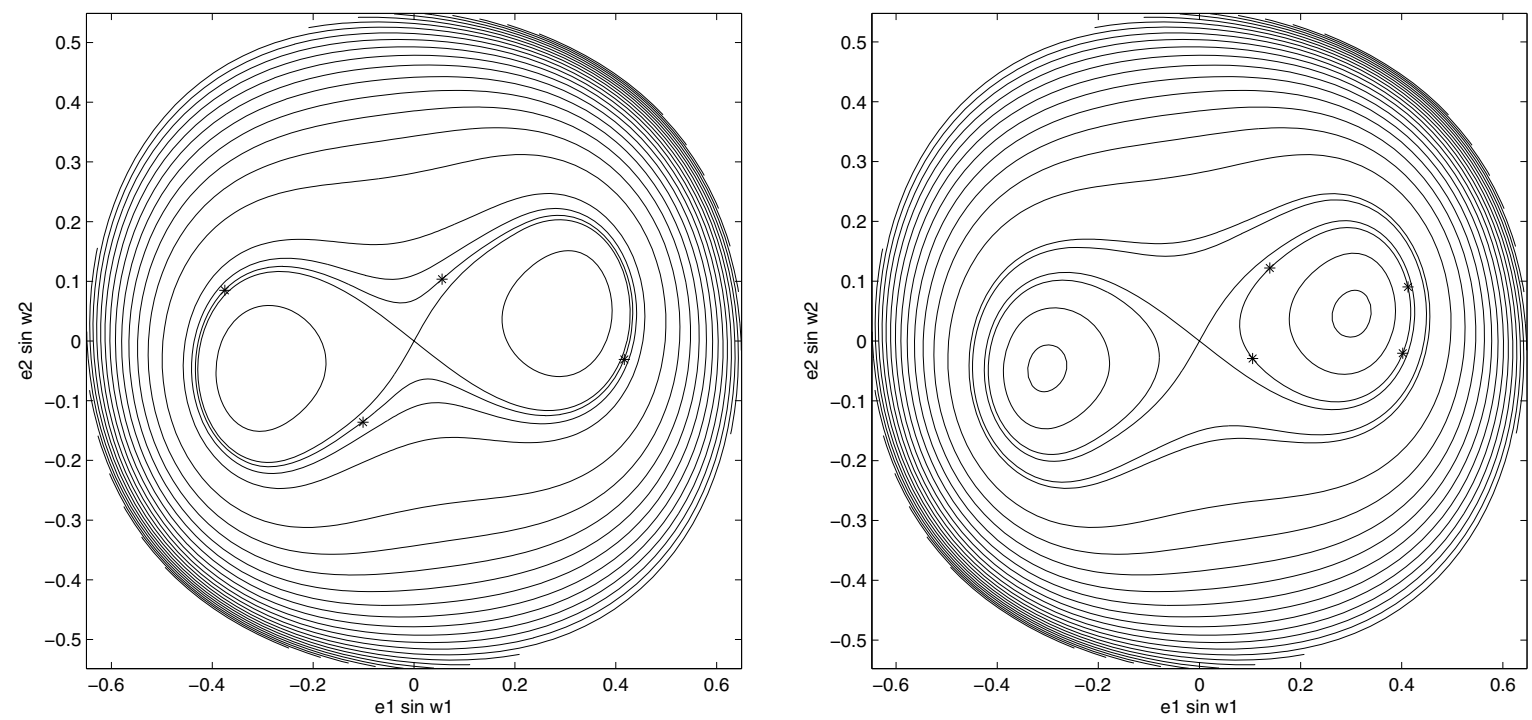

Fig. 5. Same representative planes as the right one of Fig. 4 (HD 12661 system with $i_{1}=i_{2}=30^{\circ}, I_{\text {mut }}=45^{\circ}$ ), but where the observational error on the parameter $\omega_{1}$ is taken into account: $\omega_{1}=293.7^{\circ}$ on the left and $\omega_{1}=298.9^{\circ}$ on the right.
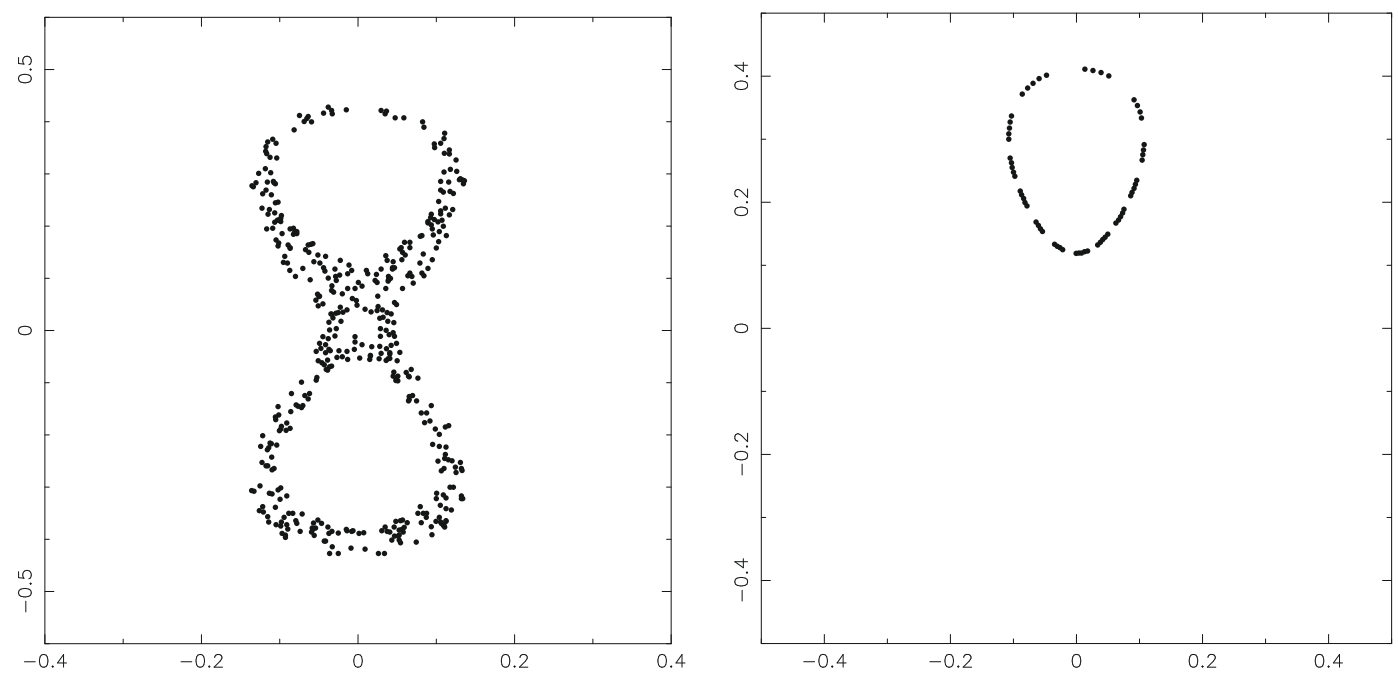

Fig. 6. Surfaces of section defined as $x_{2}=0$ with $\dot{x}_{2}<0$, projected on the plane $\left(e_{1}^{\prime} \cos w_{1}^{\prime}, e_{2}^{\prime} \sin w_{2}^{\prime}\right)$, for the same system as in Fig. 5 (HD 12661 system with $i_{1}=i_{2}=30^{\circ}, I_{\text {mut }}=45^{\circ}$ and $\omega_{1}=293.7^{\circ}$ on the left, $\omega_{1}=298.9^{\circ}$ on the right).

\section{Discussion and conclusions}

In the present work, we studied the possibility that five known extrasolar systems, composed of two major planets not in mean motion resonance, are in a stable Kozai-resonant state. The Kozai resonance offers a secular protection mechanism for systems with high mutual inclination $\left(I_{\text {mut }} \simeq 40-60^{\circ}\right)$ and large eccentricities such that, although variations of large amplitude are induced both in eccentricity and inclination, these are correlated such that the system remains stable. Hence, stable 3D systems with high eccentricities can exist, provided that they are protected by such a resonance condition.

We have undertaken a parametric study, varying the (unknown) inclinations of the orbital planes of the observed planets to the plane of the sky as well as their (unknown) nodal longitudes (which amounts to varying their masses and mutual inclination). We have shown that the systems $v$ Andromedae, HD 12661, HD 169830 and HD 74156 can be in Kozai resonance for a wide range of initial values, summarized in Table 4, provided that their mutual inclination is at least $45^{\circ}$. This means that their eccentricities and apsidal orientation are favorable for the Kozai condition to hold; namely, coupled variations of $e_{1}^{\prime}$ and $i_{1}^{\prime}$ and libration of the argument of pericenter $\omega_{1}^{\prime}$ of the inner planet, when measured in the Laplace-plane reference frame. Only the exosystem HD 155358 does not seem compatible with a Kozai behavior, as high values of $I_{\text {mut }}$ tend to destabilize the system.

Moreover, with the exception of $v$ Andromedae (80\%), only $30-50 \%$ of our initial conditions lead to chaotic motion, related to the separatrix of the Kozai resonance. This result highlights the non-negligible probability for currently detected exosystems to have high mutual inclinations. Indeed, our study has revealed stable configurations of exosystems with mutual inclinations as high as $60^{\circ}$. A few cases were found to be stable, but not related to the Kozai state. The identification of the Kozai resonance was based on the frequency decomposition criterion, introduced in Sect. 2.2. This is based on spectral characteristics that are independent of the adopted reference frame (here, the sky-plane reference frame), contrary to the well-known coupling of $e_{1}^{\prime}, i_{1}^{\prime}$ 
and $\omega_{1}^{\prime}$, which is a feature observed upon reduction of the system to the Laplace-plane reference frame.

It is important to note that throughout this study we neglected general-relativistic effects, which could be significant for the inner planets of the five systems, as is the case for binary systems (Wu \& Murray 2003; Fabrycky \& Tremaine 2007; Takeda et al. 2008) and for the "Hot Jupiter" planets (Adams \& Laughlin 2006). Moreover, we should note that these five systems may still harbor additional, yet undetected, planetary companions that could significantly modify their dynamics. Finally, as we have pointed out in Sect. 3.3, the existence or not of Kozai resonances in the selected systems can be sensitive to observational uncertainties in the orbital elements. A small change in the orbital parameters, matching the range of observational errors, can sometimes bring the system in (or out) a Kozai-resonant state. This is particularly true if we consider values of $I_{\text {mut }}$ close to the critical value $\left(\simeq 40^{\circ}-45^{\circ}\right.$, depending on the ratios of masses and semimajor axes). Thus, the observations are not yet robust enough to identify with the desired certainty extrasolar systems that are Kozai-resonant candidates. However, our study must be regarded as one that probes the possibility that secular, two-planet, exosystems are in Kozai resonance: four of the five systems studied have physical/orbital characteristics that, in $3 \mathrm{D}$, are consistent with a Kozai-resonant state, provided that the mutual inclination is between $45^{\circ}$ and $60^{\circ}$.

For these reasons, we argue that, assuming mutual inclinations in the above range, the probability for an exosystem to be in a Kozai-resonant state should not be negligible. Non-coplanar configurations of exosystems seem possible, as the preliminary results for the Upsilon Andromedae system by the HST Fine Guiding Sensor show a large mutual inclination angle $\sim 35^{\circ}$ between the orbital planes of planets $\mathrm{c}$ and $\mathrm{d}$ (McArthur et al. 2007). Such observational evidence would support the possibility of Kozai-type motion for extrasolar planets. Given that, we should ask ourselves what mechanisms, and with what efficiency, can produce systems with high mutual inclination values. In the next paragraphs we discuss several possible dynamical mechanisms.

The formation of giant planet cores by collisions of smaller embryos is a violent dynamical process characterized by orbital excitation. In such an environment, systems in secular or Kozai resonance can be formed. Such an example was given in the work of Levison et al. (1998). Thus, a possible scenario is that the Kozai resonance can be established very early in the life of a planetary system. This, in turn, raises important questions about how such 3D multi-planet systems behave in the presence of the protostellar gas nebula.

If a 2D two-planet system is embedded in a heavy gaseous disc, type II migration, combined with mean motion resonance interactions, can boost the eccentricities and inclinations of the planets to very high values, thus favoring the establishment of a Kozai resonance. This scenario has been studied by Thommes \& Lissauer (2003), where this mechanism resulted in capture in the $2 / 1$ MMR at high $I_{\text {mut }}$. Our preliminary results show that we can have a similar effect in the vicinity of $3 / 1 \mathrm{MMR}$, but not near resonances of higher order, which are consistent with the semi-major axis ratios of the systems considered in this study.

Another possible mechanism consists of planetesimal-driven migration and resonance crossing, a mechanism that is thought to have shaped the orbits of the giant planets in our solar system (Tsiganis et al. 2005). Our numerical experiments have shown that, due to the large masses of the extrasolar planets considered here (2-4 $\left.M_{\text {Jup }}\right)$, no significant migration can be induced on the planets by a "standard" planetesimals disc. Very high disc masses may be needed, which is probably not a realistic assumption.

Multi-planet scattering, caused by the previous presence of an additional planet in the system, seems to be a rather promissing mechanism. This is in fact the mechanism that is generally invoked to explain the large eccentricities observed in many exosystems (see for instance Ford et al. 2005, who studied a 2D case). Concerning the mutual inclination distribution, Chatterjee et al. (2007) reported, from their numerical planet-planet scattering simulations, quite high relative inclinations between planetary orbits (some values as high as $40^{\circ}-60^{\circ}$ ). As our first results show, 3D scattering ( starting with $e \simeq 0.001$ and $I_{\text {mut }} \simeq 0.01^{\circ}$ ) can lead to high values of $I_{\text {mut }}$, together with eccentricities that are consistent with the ones given in Table 1. The final values of $e$ and $I_{\mathrm{mut}}$ depend on the masses and relative semi-major axes of the planets. A detailed study of this mechanism is under way and statistical results will be presented in a forthcoming paper. At present, this seems to be the most promising scenario for forming 3D systems at $I_{\text {mut }}>40^{\circ}$. We note however, that the correct astrophysical picture should include a massive gaseous disc in which the planetary orbits slowly evolve and become unstable, thus leading to close encounters; otherwise, we should accept planet formation on a priori unstable orbits. We believe that soon we will be able to present our results on such a concrete scenario.

Acknowledgements. We thank the anonymous referee for her/his critical review of the first version of the manuscript and useful suggestions. We would also like to thank Benoît Noyelles for supplying his frequency-analysis code.

\section{References}

Adams, F. C., \& Laughlin, G. 2006, ApJ, 649, 992

Baluev, R. V. 2007, [arXiv: 0712.3862]

Barnes, R., Goździewski, K., \& Raymond, S. N. 2008, ApJ, 680, L57

Bean, J. L., McArthur, B. E., Benedict, G. F., \& Armstrong, A. 2008, ApJ, 672, 1202

Brouwer, D., \& Clemence, G. M. 1961, Methods of celestial mechanics (New York: Academic Press)

Butler, R. P., Wright, J. T., Marcy, W., et al. 2006, ApJ, 646, 505

Champenois, S. 1998, Thesis, Observatoire de Paris

Chatterjee, S., Ford, E. B., Matsumura, S., \& Rasio, F. A. 2007 [arXiv: astro-ph/0703166]

Chiang, E. I., Tabachnik, S., \& Tremaine, S. 2001, AJ, 122, 1607

Fabrycky, D., \& Tremaine, S. 2007, ApJ, 669, 1298

Ford, E. B., Lystad, V., \& Rasio, F. A. 2005, Nature, 434, 873

Harrington, J., Hansen, B. M., Luszcz, S. H., et al. 2006, Science, 314, 623

Jacobi, C. G. J. 1842, Astron. Nachr., 20, 81

Jefferys, W. H., \& Moser, J. 1966, AJ, 71, 568

Kozai, Y. 1962, AJ, 67, 591

Laskar, J. 1990, Systèmes de Variables et Éléments, in Les Méthodes modernes de la Mécanique Céleste, ed. D. Benest, \& C. Froeschlé (éditions Frontières), 63

Laskar, J. 1993, Physica D, 67, 257

Laskar, J. 1997, A\&A, 317, L75

Levison, H. F., \& Duncan, M. J. 1994, Icarus, 108, 18

Levison, H. F., Lissauer, J. J., \& Duncan, M. J. 1998, AJ, 116, 1998

Libert, A.-S., \& Henrard, J. 2007, Icarus, 191, 469

Libert, A.-S., \& Henrard, J. 2008, Celest. Mech. \& Dyn. Astro, 100, 209

McArthur, B., Benedict, G. F., Bean, J. \& Martioli, E. 2007, BAAS, 211, 134.17

Michtchenko, T. A., Ferraz-Mello, S., \& Beaugé, C. 2006, Icarus, 181, 555

Murray, C. D., \& Dermott, S. F. 1999, Solar System Dynamics (Cambridge University Press)

Poincaré, H. 1892, Méthodes nouvelles de la mécanique céleste (GauthierVillars, Paris), reprinted by Blanchard (1987)

Robutel, P. 1995, Celest. Mech. \& Dyn. Astro, 62, 219

Stepinski, T., Malhotra, R., \& Black, D. 2000, ApJ, 545, 1044

Takeda, G., Kita, R., \& Rasio, F. A. 2008, ApJ, 683, 1063

Thommes, E. W., \& Lissauer, J. J. 2003, ApJ, 597, 566

Tsiganis, K., Gomes, R., Morbidelli, A., \& Levison, H. F. 2005, Nature, 435, 459

Wisdom, J., \& Holman, M. 1991, AJ, 102, 1528

Wu, Y., \& Murray, N. 2003, ApJ, 589, 605 